\title{
Uncovering the Mistford Toxic Conspiracy
}

\author{
Dirk Streeb* \\ Universität Konstanz \\ $\begin{array}{cc}\text { Juri Buchmüller }^{\dagger} & \begin{array}{l}\text { Udo Schlege } \\ \text { Universität Konstanz }\end{array} \\ \text { Universität Konstanz }\end{array}$ \\ Wolfgang Jentner ${ }^{\S}$ \\ Universität Konstanz \\ Michael Behrisch ${ }^{\text {Il }}$ \\ Harvard University \\ Bruno Schneider" \\ Daniel Seebacher** \\ Universität Konstanz \\ Universität Konstanz
}

\begin{abstract}
To help ornithologist Mitch in understanding the poor development of the Rose-crested Blue Pipit in terms of the VAST Challenge 2017 Grand Challenge, we apply a diverse set of custom specialized tools and out-of-the-box data analysis systems to a rich data set consisting of satellite images, gas sensor measurements, movement traces and newsletter issues. Following the Visual Analytics approach, we implement a collaborative analysis loop and are able to combine data and gain insights into the current situation of the Boonsong Lekagul Nature Preserve. Finally, we come up with a hypothesis that combines suspect observations to a coherent story of illegal disposal of toxic waste involving two companies located in the reserve's vicinity.
\end{abstract}

\section{INTRODUCTION}

Why do the Rose-crested Blue Pipits in the Boonsong Lekagul Nature Preserve disappear? is the central question of this year's VAST Challenge. In order to come up with an answer featuring latent as well as acute factors, we undertake an analysis of the diverse data provided. This data includes movement traces of all cars and trucks in the reserve for thirteen months and six-channel satellite imagery of twelve days across three years. Furthermore, there are gas sensor data for four chemical substances and wind measurements of three months, and additionally eight issues of a newsletter including four years of stock prices of local companies.

Here, we describe our approach to solving the mystery around the declining bird population. Then, we summarize our insights on general threats to wildlife in the preserve and investigate the events connected to the suspected illegal disposal of toxic waste more deeply. Finally, we present next steps that the park management should consider in order to save the birds and other wildlife.

\section{Analysis Approach}

As the given data is very diverse, contains missing values, and overlaps only partially in time, we are not able to apply a fully automated analysis. On the other hand, the movement and sensor data sets are too large to be processed efficiently by humans alone. Therefore, we implement a Visual Analytics approach combining automated, e.g., movement sequence mining, and manual analysis using data visualization, e.g., stock data extraction and analysis. To begin with, we combine results gathered in the three Mini-Challenges. In an iterative and collaborative analysis loop [2] we integrate combined

\footnotetext{
*e-mail: streeb@dbvis.inf.uni-konstanz.de

†e-mail: buchmueller@dbvis.inf.uni-konstanz.de

¥e-mail: udo.3.schlegel@uni-konstanz.de

§e-mail: jentner@dbvis.inf.uni-konstanz.de

Ile-mail: behrisch@g.harvard.edu

"e-mail: schneider@dbvis.inf.uni-konstanz.de

**e-mail: seebacher@dbvis.inf.uni-konstanz.de
}

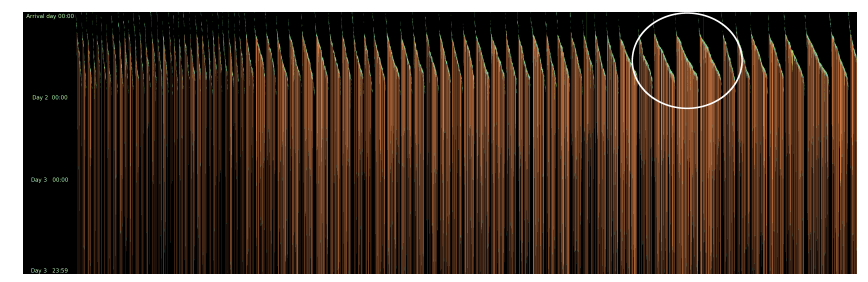

Figure 1: Seasonal traffic increases in spring and during weekends peaking on the weekend of the $4^{\text {th }}$ of July (circled). Each column represents one vehicle. Columns are aligned by time of day and ordered by day of year.

results, come up with new hypotheses, and investigate these further across all available data sets.

On the software side we use a toolbox of diverse applications. The toolbox includes specialized software created for the MiniChallenges (e.g., shown in Figures 2 and 3), e.g., SIZE: Satellite Image Zooming and Exploration [same issue], as well as standard third-party software like KNIME [1] and Tableau. Further, we are able to reuse the Visual Movement Explorer we developed for the 2015 VAST Challenge with minor modifications [3] (see Fig. 1).

We use these tools in parallel and collaboratively connect clues between data sets. For example, we are able to compare spatial findings gain from trip sequence mining with geographical features shown in the satellite images. As a result, we manage to connect clues to a timeline and try to find missing pieces and investigate unexpected co-occurrences.

The loose structure of our toolbox offers the flexibility we need to solve a difficult, loosely defined task in a short time. First, implementation time of separated components can be optimized such that we can start to analyze some data early on. Second, parallel use of multiple tools provides sufficiently many opportunities to integrate the data that by its diverse nature would be difficult to integrate on the machine side. Finally, it is easy to extend the focused tools or add new tools to the toolbox whenever new demands arise.

\section{Threats to WiLdLife}

We identify a high-level of traffic, land use and pollution as major threats to wildlife in the reserve. The main part of traffic in the park consists of a large amount of seasonal tourists (see Fig. 1). These stay in the park for a long season from spring through fall and bring noise, motion as well as litter to the reserve.

Remaining regular traffic includes a considerable amount of heavy traffic. Both trucks and buses pass through the park all day long. Especially the large amount of traffic at night might put stress on wildlife. Further, all kinds of motorized traffic contribute to the pollution of the park with their exhausts.

A second threat to wildlife becomes visible in the satellite images. Land use within the park increases over the measured period. For example, a road close to the center of the park is widened and all vegetation is removed from some space next to it. Henceforth, the habitat actually available to wildlife shrinks while the bounds and size of the reserve remain the same on maps. 


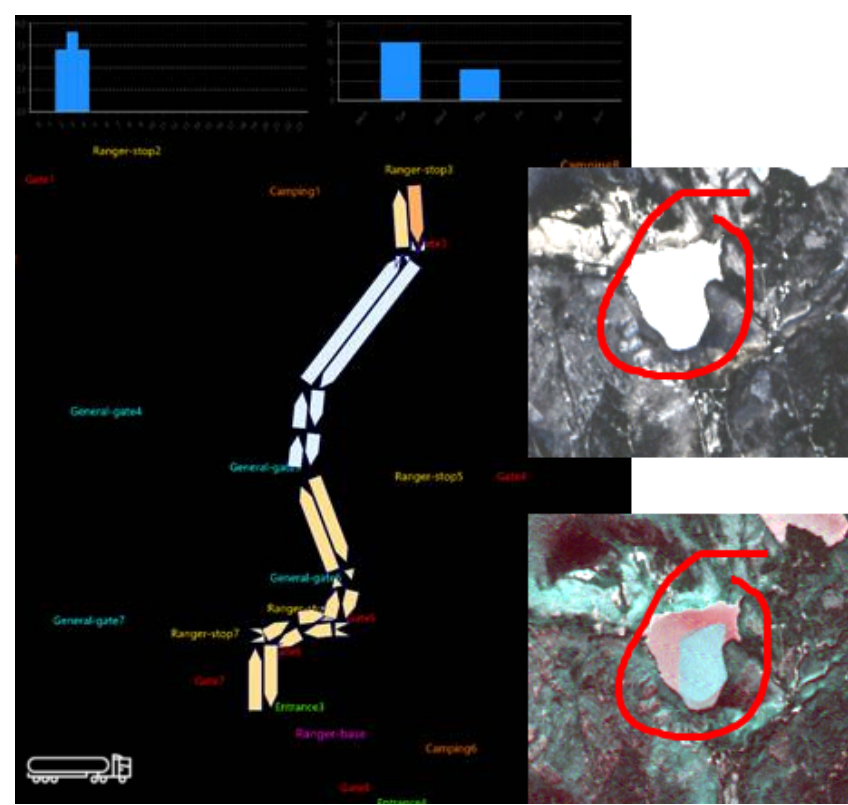

Figure 2: On Tuesday and Thursday early mornings four axle trucks drive from the entrance at the Mistford industrial park to a place near a river floating in the lake. Later, the lake changes its appearance (see insets). Thus, we suspect that illegally disposed waste pollutes the lake.

As a last, highly influential, factor we consider intentional pollution at an industrial scale. In the case of the Lekagul preserve, pollution is brought into the reserve along two paths. On the one hand, wind drifts polluted air from outside the park into it. On the other hand, trucks bring toxic waste inside the park and dispose it there illegally. The next section goes into detail on this matter.

Taken together, we expect the combination of a generally high level of stress induced by constantly high levels of traffic, the destruction of habitats and the acute pollution to pose a combined threat to wildlife.

\section{Suspicious Behavior}

While common behavior as described in the previous section can have negative effects on wildlife there seem to happen more harmful things in Mistford Industrial Park and the reserve next to it. While authorities implemented stricter emission laws in 2013, it looks like some companies circumvent these illegally. To begin with, there are regular tours of heavy trucks from the Mistford industrial park to a remote non-public place in the reserve on early Tuesday and Thursday mornings while rangers are out of service. Within two years a lake nearby changes its appearance as if polluted from the side the trucks go to (See Fig. 2).

Additionally, the gas measurement system, which authorities use to observe violations against emission laws, shows signs of sabotage. One sensor for hazardous Methylosmolene shows a systematic pattern of malfunction producing mislabeled records. Interestingly, this only occurs when the wind blows from south-westerly directions (See Fig. 3). Therefore, we suspect sabotage by a company located to the west of the sensor station as all other sensor stations are positioned in a semi-circle to the west of the industrial park.

Combining these two pieces of evidence with information presented in the newsletter, we come up with the following suspicion: In early 2014 both companies, Kasios and Radiance, get in touch more closely in the newly established Company Sustainability Champion (CSC) program. Instead of honestly trying to improve their production for environmental benefit, they begin to exchange infor-

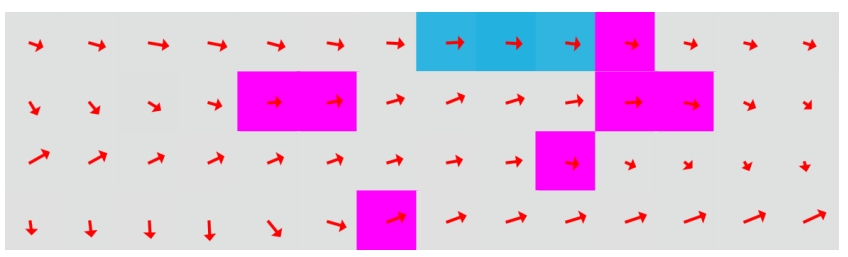

Figure 3: Missing (magenta) and high (blue) measurements of toxic gas only appear on south-westerly winds (arrows). Due to this strong correlation and the high amount of missing values we suspect sabotage.

mation about issues with meeting environmental restrictions. Kasios, who suffered more from the strict regulations, proposed Radiance to solve their problem of getting rid of toxic waste from Radiance's new spray paint to be introduced in early 2015 . In exchange, Radiance manipulates the sensor for Methylosmolene of gas measurement station 6, which happens to be located on their premises.

Following their conspiracy, Kasios begins to pick up Radiance's waste and dispose it at night at ranger-stop3 in the reserve. On the other hand, Radiance manipulates the sensor on request of Kasios when there are south-westerly winds. As a result, Kasios can exhaust large amounts of Methylosmolene that drift to the east without obviously showing up in any sensor records as all other sensors are positioned in a semi-circle towards the west and the values of sensor 6 are mislabeled.

Kasios can implement their expansion plans from 2014 through 2017 just like Radiance who financed their new production line financed by a stock split. Both companies profit from this conspiracy and their stock prices rise quickly.

In order to test this theory, we would need access to the companies' bookkeeping. Additionally, our evidence could be improved by taking water samples from the lake that we expect to be polluted and checking the sensor station for signs of manipulation. Overall, we are confident that at least one of the two companies is involved in some illegal behavior harmful to the environment.

\section{CONCLUSION}

Taken together, we find changes in the habitat of the Rose-crested Blue Pipits, which appear to be human-made. Most importantly, we identify illegal behavior within and in close proximity to the Boonsong Lekagul Nature Preserve and point out suspects for further investigation. Finally, we suggest measures to improve the situation of the natural environment in general and the Rose-crested Blue Pipit in particular.

Throughout all these steps we engage in a combined approach of human-centered and machine-driven data analysis following a Visual Analytics process. Thus, we are able to provide ornithologist Mitch with hypotheses to investigate further and a set of data analysis tools to get the most out of the data he has access to.

\section{REFERENCES}

[1] M. R. Berthold, N. Cebron, F. Dill, T. R. Gabriel, T. Kötter, T. Meinl, P. Ohl, C. Sieb, K. Thiel, and B. Wiswedel. KNIME: The Konstanz Information Miner. In Studies in Classification, Data Analysis, and Knowledge Organization (GfKL 2007). Springer, 2007.

[2] D. Sacha, A. Stoffel, F. Stoffel, B. C. Kwon, G. Ellis, and D. A. Keim. Knowledge Generation Model for Visual Analytics. IEEE Transactions on Visualization and Computer Graphics (Proceedings Visual Analytics Science and Technology 2014), 20(12):1604 - 1613, 2014. doi: 10. 1109/TVCG.2014.2346481

[3] D. Streeb, U. Schlegel, J. Buchmüller, F. Fischer, and D. A. Keim. Using Visual Analytics to Analyze Movement and Action Patterns. In IEEE Conference on Visual Analytics Science and Technology (VAST Challenge 2015 MC1), 2015. doi: 10.1109/VAST.2015.7347665 